\title{
Undescended testes in children: the paediatric urologist's point of view
}

\author{
Pierre D E Mouriquand \\ Department of Paediatric Urology, Hôpital Mère-Enfants, Claude-Bernard University, Lyon 1, 59, Boulevard Pinel, 69500 Bron, France \\ (Correspondence should be addressed to P D E Mouriquand; Email: pierre.mouriquand@chu-lyon.fr)
}

\begin{abstract}
Undescended testes (UDT) are found in 1\% boys at the age of 1-year old, $3 \%$ of full-term male infants and 33\% in premature babies at birth. Spontaneous descent is possible until 6 months of age. The two main phases of descent of the testis during gestation are described, as well as the possible aetiologies, the consequences of an abnormal migration, and the main locations of UDTs. Clinical examination is essential and should be done in optimal conditions. Laparoscopic exploration is the only valuable complementary investigation in the case of non-palpable UDT.
\end{abstract}

European Journal of Endocrinology 159 S83-S86

Four main situations should be distinguished:

i) Isolated unilateral palpable undescended testes (UDT) without associated genital anomalies, which requires a straightforward orchidopexy without other investigations

ii) UDT with associated genital anomalies, which requires a genetic and endocrine assessment

iii) Unilateral non-palpable UDT that requires a laparoscopic exploration that will determine whether there is a testis, if it is preservable and if it can be brought down in one or two steps

iv) Bilateral non-palpable testes that should raise three questions:

- Is this child a boy? Karyotype; $170 \mathrm{OH}$ progesterone

- Are the testes present? human chorionic gonadotrophin (HCG) test/anti-Müllerian hormone (AMH)/follicle-stimulating hormone-luteinizing hormone (FSH-LH)

- Where are the testes? Laparoscopy: one- or two-stage orchidopexy.

Differential diagnosis is the retractile testes, anorchidy and congenital adrenal hyperplasia $(\mathrm{CAH})$. The issue of the ascending testis is approached.

Consequences of UDTs on testicular development, fertility and tumours are discussed as well as the various therapeutic options and the follow-up.

This paper was presented at the 5th Ferring International Paediatric Endocrinology Symposium, Baveno, Italy (2008). Ferring Pharmaceuticals has supported the publication of these proceedings.

\section{UDT in children: the paediatric urologist point of view}

The UDT is the most common condition met by paediatric urologists. It is classically found in $1 \%$ of boys at the age of 1 year, in 3\% of full-term male infants and in one-third of premature babies at birth $(1,2)$. Although mostly diagnosed during the neonatal period, UDT can be found much later in life and are then called 'ascending' testes or 'acquired' UDT.

The aetiologies of UDT are essentially unknown, although various possible mechanisms have been reported: abnormal migration of the gubernaculum testis, deficient hormonal secretion, persistent processus vaginalis, abnormal abdominal wall (prune belly syndrome), congenital neurological anomalies (spina bifida) and genetic anomalies $(1,2)$.

The mechanisms of descent of the testes are complex and partially unknown. They classically involve two phases. The first one is the abdominal migration that happens between the 8th and 15th week of gestation and which is under the possible control of AMH, Leydig-derived insulin-like peptide (INSL3) and androgens. It is likely that the gubernaculum testis and the cranial suspensory ligament are the main targets of these agents. The second phase of the testicular migration is inguinoscrotal and occurs between the 28th and 35th week of gestation. Androgens and calcitonin gene-related peptide may have an action on the spinal nucleus of the genitofemoral nerve that itself innervates the gubernaculum testis. These hypotheses mainly come from the rat model and cannot be fully extrapolated to the human model (1-3).

Whatever mechanism is involved in the failed descent of the testes, the main consequence of a malpositioned 
testis is its tissular maldevelopment. One of the possible factors of tissular maldevelopment of the UDT is the intra-abdominal temperature that may lead to an abnormal differentiation of the gonocytes into $\mathrm{A}$ spermatogonium.

\section{Diagnosis of UDT}

Clinical examination is crucial and should be done in optimal conditions requiring a calm child laying down on his back, warm hands and patience. If these conditions are not met, no definite conclusions can be drawn and the examination should be repeated later. It is quite common to get referrals from practitioners who could not feel a testis where no anomaly can be found when another examination is performed. It is also classical to decide an orchidopexy and find two testes sitting perfectly normally in each scrotum when the child is under general anaesthetic. One can never insist enough on the modalities of clinical examination, which need to be taught to each medical student and should be able to answer the following questions: are the testes palpable? If yes, what are their size, situation and consistence?

Clinical examination finds that $80 \%$ of UDT are palpable and sit in the inguinal superficial pouch (30\%), the inguinal canal (20\%), the upper scrotum (45\%) and rarely $(5 \%)$ the perineum or the thigh and that $20 \%$ of UDT are non-palpable and sit in the abdominal cavity $(1,2)$. At the end of a careful clinical examination, one should be able to say whether the testicle is palpable, non-palpable or if it is unknown. This leads to four different situations and four different managements:

i) The child presents with an isolated unilateral palpable testis without associated genital anomalies. Surgery should then be indicated without any other complementary investigations.

ii) The UDT (uni- or bilateral; palpable or not) is associated with other genital anomalies, essentially micropenis and hypospadias: the child needs genetic and endocrine assessments followed by surgery.

iii) The UDT is not palpable without associated genital anomalies: ultrasound scans in UDT are controversial as they can demonstrate an intra abdominal testis in less than $50 \%$ cases (5). The only reliable complementary investigation is laparoscopy, which should be able to answer three questions: Is there a testis? Should it be kept? If no, it should be removed laparoscopically; if yes, can the testis be brought down into the scrotum with a one- or two-stage orchidopexy.

iv) Both testes are non-palpable (4). Three questions should be answered. First, is this child a boy? The karyotype will answer this question. If the child is $46, \mathrm{XX}$, the most frequent diagnosis is $\mathrm{CAH}$ and measurement of $17-\mathrm{OH}$ progesterone level confirms the diagnosis. It is actually rare that the genital tubercle looks fully masculine in a CAH girl. If the child is 46 , $\mathrm{XY}$, the next question is, are the testes present? An HCG test, a measurement of AMH, FSH and $\mathrm{LH}$ levels should answer this question. If there is no testicular response, it is most unlikely that there is some testicular tissue sitting in this child. We would not therefore recommend any surgical exploration at this stage. If these tests show that there is a testicular response, then the third question is, where are these testes? Laparoscopy will tell and will also help to choose the best surgical option.

Beside the situation of the $\mathrm{CAH}$ girl, two other differential diagnoses need to be identified. The retractile testes are due to an active cremasteric reflex. Here again, a careful clinical examination is required to distinguish a retractile testis from a UDT. Retractile testes can be easily milked down to the bottom of the scrotum where it should stay for a few seconds when the examiner stops pulling on the gonad. For years, retractile testes have been considered as a normal variant, but it seems more and more accepted that retractile testes may evolve to a proper UDT at a later age of life. It is probably in this category of patients that 'ascending' testis or 'acquired' UDT are found. Approximately, one-third of acquired testes become UDT at a later of life. It is therefore recommended to examine these children on an annual basis $(6,7)$.

\section{Evolution of UDT}

A UDT can meet four main complications:

i) Atrophy and necrosis are found in 3\% of nonoperated UDT. Two factors are involved in the disappearance of these testes. The first one is poor quality of the testicular tissue and it is accepted that the higher the testicle sits in the abdomen the more dysgenetic it is. Dysgenetic testes may involute. The second one is the presence of a patent processus vaginalis, i.e. an inguinal hernia in most UDTs. If the hernial sac is full, it may compress the spermatic vessels causing a testicular necrosis.

ii) Fertility is a well-reported consequence of UDT. It is actually a controversial topic. One should first remember that between 15 and 20\% of 'normal' couples are infertile, i.e. couples with a male with two normally descended testes. Australian studies show that $60-80 \%$ of fathers who had a unilateral UDT have children (almost like the general population) against $40-50 \%$ when both testicles have been descended. In this latter group, 
$75 \%$ of spermograms are abnormal. An elevated FSH is a criterion of poor prognosis. It is actually unclear whether the age of orchidopexy matters in the fertility outcome, although it is reported that histological damage of the testicle is more common after the age of 2 years. This probably explains why the recommended window for treatment of UDT is usually between 6 months and 2 years of age, knowing that a spontaneous testicular migration is theoretically possible during the first 6 months of life.

iii) Testicular tumours are most frequent in adults who have a history of UDT. It is classically reported that the risk is increased by $20-50 \%$ but here again this is a controversial issue. UDT have a 1.5-2\% risk of developing a tumour at an adult age. Surgery does not seem to reduce the risk of tumour but makes the testicle palpable i.e. easier to examine by the patient himself or a urologist. The age at surgery has recently been pointed out as a relevant factor in the potential risk of testicular tumours (8). This study reports a cutting age of 13 years, over which the risk of testicular cancer is higher. However, the vast majority of UDT are treated before the age of 2 years, so further studies are needed before stating whether the surgery has any role to reduce this risk.

iv) The association of UDT and carcinoma in situ (CIS) has been reported although its meaning is also controversial. CIS reflects the abnormal presence of foetal cells that did not reach the stage of type A spermatogonia and may grow on their own (9).

v) Trauma and torsion of UDT are classically more common as the testicular attachments of UDT are looser than in normally descended testes. One should be aware that acute abdominal pain and an empty scrotum could be related to the torsion of a UDT.

\section{Treatments of UDT}

The aim of the treatments of UDT is to bring the testis into the ipsilateral scrotum before the age of 2 years to preserve the endocrine and exocrine testicular functions. A spontaneous descent of a UDT is possible before 6 months of age and histological damages of the testis seem to be permanent after 2 years of age (1). This is the reason why most paediatric urologists would recommend surgery between 6 and 24 months of age. However, the surgical trauma caused on the spermatic vessels and the vas deferens by the dissection of the spermatic cord in young infants compared with older children needs to be evaluated.

The methods to achieve these aims are medical or surgical. The medical means mainly involve the use of BHCG and/or LH-releasing hormone (LHRH), whereas surgical techniques mainly involve one- or two-stage orchidopexy using open or laparoscopic procedures, and orchidectomy when the testicular tissue is not preservable.

Hormonal treatments are detailed elsewhere; various protocols have been reported using BHCG, LHRH and a combination of these two. There is no consensus on the therapeutic effects of this hormonal stimulation and its long-term consequences are not clearly established. For some, HCG treatment may impair the histology of the seminiferous tubules (10) in the rat testes, whereas other studies demonstrated that the number of spermatogonia per tubule is increased or unchanged after HCG. Positive effect of HCG stimulation on testicular descent is extremely variable in the literature from 6 to $75 \%$. The place of recombinant gonadotrophins remains to be defined. BHCG is also used by some as a preparation to surgery in case of redo procedure or high non-palpable testes. It increases the blood supply of the testis and facilitates the surgical procedure.

Surgical treatments are well established. The classical inguinal approach of UDT implies the opening of the inguinal canal, the dissection of the spermatic cord, the ligation of the patent processus vaginalis that is frequently present and the placement of the testis in the so-called Dartos pouch, a space sitting between the scrotal skin and the underlying smooth muscle layer. To achieve a satisfactory surgical descent of the UDT, i.e. to get enough length of the cord to bring the testicle down without tension, one or two of the three vascular supplies of the testicle need to be divided. The testes have three blood supplies: the spermatic vessels, the main blood supply, which is divided in the Fowler-Stephens procedure; the funicular vessels that accompany the vas deferens; and the gubernacular vessels that are divided in the classical one-stage orchidopexy.

Laparoscopy is a revolution as it has two main roles, diagnostic and therapeutic. Laparoscopy allows identification of the gonad, its position and its quality. It also has a major role in the first stage of a two-stage orchidopexy or in orchidectomy.

This surgery should be exclusively performed by paediatric urologists or paediatric surgeons in infants and children.

Whichever procedure is chosen to bring the testicle down, these patients need a careful follow-up involving regular self-palpation of the testes after puberty to detect any irregularity related to a testicular tumour. In case of an abnormal palpation of the testis, the patient should be examined by a urologist who will perform a clinical and ultrasound examination of the testes and a measurement of the plasma tumour markers (BHCG and alpha foeto protein). Some adolescents may request the implantation of a testicular prosthesis to replace one or two missing testicles. Late orchidopexy remains a controversial issue although the current tendency is to be conservative and keep these testicles with the usual advice of a cautious follow-up. 


\section{Disclosure}

This paper forms part of a European Journal of Endocrinology supplement, supported by Ferring Pharmaceuticals. The author discloses no potential conflicting relationship with Ferring. This article was subject to rigorous peer review before acceptance and publication.

\section{References}

1 Hutson JM. Undescended testes. In Pediatric Surgery and Urology: Long-Term Outcomes, edn 2, ch. 51, pp 652-663. Eds MD Stringer, KT Oldham \& PDE Mouriquand, Cambridge: Cambridge University Press, 2006

2 Baker LA, Silver RI \& Docimo SG. Cryptorchidism. In Pediatric Urology, edn 1, ch. 46, pp 738-753. Eds J Gearhart, R Rink \& PDE Mouriquand, Philadelphia: W.B. Saunders, 2001.

3 Tomiyama H, Sasaki Y, Huynh J, Yong E, Ting A \& Hutson JM. Testicular descent and inguinal hernia: the Melbourne perspective. Journal of Pediatric Urology 20051 11-25.

4 Quimby GF \& Mouriquand PDE. In Case Presentations for the MRCS and AFRCS, Bilateral Undescended Testes, Vol 2, ch 24, pp 122-126. Eds PH Hornick, J Lumley \& P Grace, Oxford: Butterworth Heinemann, 1997.
5 Nijs SM, Eijbouts SW, Madern GC, Leyman PM, Lequin MH \& Hazebroek FW. Non palpable testes: is there a relationship between ultrasonographic and operative findings. Pediatric Radiology 2007 37 374-379.

6 Hack WW, Sijstermans K, van Djik J, van der Voort-Doedens LM, de Kok ME \& Hobbelt-Stoker MJ. Prevalence of acquired undescended testis in 6 year, 9 year and 13 year old Dutch schoolboys. Archives of Disease in Childhood 200792 17-20.

7 Mouriquand P. The nomad testis. Archives of Disease in Childhood 2007923.

8 Pettersson A, Richiardi L, Nordenskjold A, Kaijser M \& Akre O. Age at surgery for undescended testis and risk of testicular cancer. New England Journal of Medicine 2007356 1835-1837.

9 Sonne SB, Kristensen DM, Novotny GW, Olesen IA, Nielsen JE, Skakkebaek NE, Rajpert-De Meyts E \& Leffers H. Testicular dysgenesis syndrome and the origin of carcinoma in situ testis. International Journal of Andrology 200831 275-287.

10 Kaya C, Karaman MI, Pirincci N, Ozturk M \& Yilmazgumrukcu G. Human chorionic gonadotropin deteriorates the histology of rat testes. Urologia Internationalis $2006 \mathbf{7 6} 274-277$.

Received 3 July 2008

Accepted 8 July 2008 